\title{
A MULTIMEDIA-BASED GUIDANCE SYSTEM FOR VARIOUS CONSUMER DEVICES
}

\author{
M. Kropfberger, R. Tusch, M. Jakab, J. Köpke, M. Ofner \\ M3-Systems Research Lab, Department of Information Technology, University Klagenfurt, Austria* \\ \{Michael.Kropfberger, Roland.Tusch, Michael.Jakab, Julius.Koepke, Michael.Ofner\}@m3-system.com \\ H. Hellwagner, L. Böszörmenyi \\ Department of Information Technology, University Klagenfurt, Austria \\ \{Hermann.Hellwagner, Laszlo.Boeszoermenyi\}@uni-klu.ac.at
}

Keywords: Multimedia-based guide for stationary and mobile devices, context-awareness, content adaptation, presentation adaptation, content management.

\begin{abstract}
This paper introduces a Web-based guidance system which supports optimized presentations of sights or exhibited objects on different types of available stationary and mobile consumer devices, possibly running different operating systems. This is accomplished by adapting both the objects' content, as well as their presentation to the current usage context. Content thereby may be encoded in different presentation formats like video, audio, image, and marked-up text. The usage context embraces a set of properties describing the current usage environment of the guide. This includes, for example, the consumer device's capabilities, its current location, and the user's preferences. Both, the content adaptation and presentation services are based on standard Web technologies for increased interoperability. Finally, the guidance system is augmented with a Web-based content management and a statistics module, which enable for remote content administration and usage evaluations, respectively.
\end{abstract}

\section{INTRODUCTION}

With the emergence of affordable hand-held consumer devices, offering adequate screen resolutions and enough CPU power for reasonable video decoding, multimedia services are no more limited to powerful stationary devices. Many of them are becoming feasible to such mobile devices. Due to the mobility factor and battery operating times of over three hours, such a service may be a multimedia-enhanced mobile guide, which, for example, is used in exhibitions or touristy regions. Thereby, the guidance may be supported by context-sensitive usage information which is obtained in advance and/or dynamically during the guiding process.

This paper introduces a Web-based guidance system, called M3-Guide in the sequel, which supports optimized presentations of exhibited objects or places

${ }^{*}$ This work was partially supported by the Austrian Science Fund (FWF) under project L92-N13 (CAMUS: Context-Aware Multimedia Services), and by the austrian network for e-tourism (anet) under project Tourguide für Web und mobile Endgeräte. of interest on different available stationary and mobile consumer devices. It does this by adapting both the objects' content, as well as their presentation to the current usage context. Content thereby may be coded in different presentation formats like video, audio, image, and marked-up text. The usage context embraces a set of properties describing the current usage environment of the guide, including user profile, device profile, network profile, location, and time.

\section{RELATED WORK}

Context-awareness has become a major issue in mobile computing recently. Especially for mobile devices with limited device capabilities (like screen resolution, network bandwidth, and computing power), content and its presentation often need to be tailored to meet these limitations. This is essentially required for multimedia presentations which are mostly encoded for high screen resolutions with high bit rates. A number of research contributions address adaptation methods and techniques for content and pre- 
sentation adaptation. For example, adaptation services for multimedia content are addressed by the QBIX intelligent media proxy (Schojer et al., 2003; Kropfberger and Hellwagner, 2005), the Princess platform for multimedia service adaptation (Korva et al., 2001), the Negotiation and Adaptation Core (NAC) architecture (Lemlouma and Layada, 2004), the MM4U framework for personalized multimedia content (Scherp and Boll, 2005), and especially within the scope of MPEG-21 Digital Item Adaptation (Bormans and Hill, 2002; Vetro et al., 2006; Devillers et al., 2005; Vetro and Timmerer, 2005; Timmerer and Hellwagner, 2005). Web-based presentation adaptation relying on various markup languages like HTML, XHTML and SMIL is a quite mature research topic, extensively addressed in the area of adaptive hypermedia systems (Brusilovsky, 1996; Bra and Calvi, 1998; Bulterman et al., 2005; Lemlouma and Layada, 2003).

All adaptation methods have in common that they take into account context information in their adaptation steps. The context describes the usage environment of the application and spans a number of dimensions. These include the user's profile (e.g., language, age class, interests), the device's profile (e.g., screen resolution, decoding capabilities), the network's profile (e.g., available bit rate, delay), and the location and time of content consumption. Context information may be partially described by the Universal Profiling Schema (Lemlouma and Layada, 2004), which is based on CC/PP and RDF (Klyne et al., 2004), by UAProf descriptors for device capability descriptions (Open Mobile Alliance, 2006), and by MPEG21 Usage Environment Description tools of MPEG21's Part 7 Digital Item Adaptation (Bormans and Hill, 2002).

There are already guidance systems for hand-held mobile devices which take into account some dimensions of usage context. Two examples of such guides are the Cyberguide (Abowd et al., 1997) and the MobiDENK (Krösche et al., 2004) guide. However, they cover only the two dimensions user profile and location, but they do not take into account device and network capabilities, and hence they do not adapt content and presentations dynamically to different networked and non-networked stationary and mobile consumer devices.

\section{GENERAL OVERVIEW}

Figure 1 provides a general overview of the M3Guide. On the left-hand side it is shown that content of various types (video, audio, text, image) for objects of interest can be fed into the system via a Web-based and hereby platform-independent content management system (CMS). Content may be stored in different languages, and associated with predefined special user profiles like adults, children, historical insterest, technical interest, and many more. The Webbased CMS is accessible from everywhere, and the content may be managed in parallel by different responsible persons.

Once fed into the system, the content is then adapted and presented according to the current usage context. This includes the location either automatically determined by different technologies like GPS and Bluetooth (Santner et al., 2006), or manually captured by entering the number of the current object of interest. The M3-Guide also adheres to the user profile containing information about her/his age class, preferred language, and interests. And finally, content may be presented depending on the current time. Thus, messages like "dear visitor, the park is closing in a few minutes" are displayed right on time.

One major focus of the M3-Guide is the large variety of networked or disconnected consumer devices. Networked devices may be wireless mobile phones using UMTS, and WLAN-connected Pocket PCs, laptops and Tablet PCs. Stationary devices may be strategically positioned info terminals, private Web PCs, or video walls. These networked devices receive all content directly from our system, which optimizes it to the current usage context. This is illustrated by the centralized adaptation module. Video and audio streams are adapted to fit to locally available codecs and CPU-bearable frame and bit rates. Images are resized and texts are prepared to fit the screens and available fonts. Further, the whole HTML-based browser application including navigation, search forms, and icons is adapted to match the devices' capabilities.

Since these devices are usually connected, the content is up-to-date and content changes in the CMS are immediately forwarded to the presenting devices. On the other hand, the CMS is capable of producing so called "local dumps" for disconnected hand-held devices. Such dumps can be later stored on memory cards. Another local dump can be generated for data DVDs, which may be used as give-away DVDs for customers. The DVD presentation is optimized for high screen resolution and high audio and video qualities. These two dumps also perform auto-starts when either the memory card or the DVD is inserted into a corresponding device. A local dump can also be created for printable media, where a list of all objects of interest with printable content (images and texts, but obviously no audio and video) is prepared to allow 


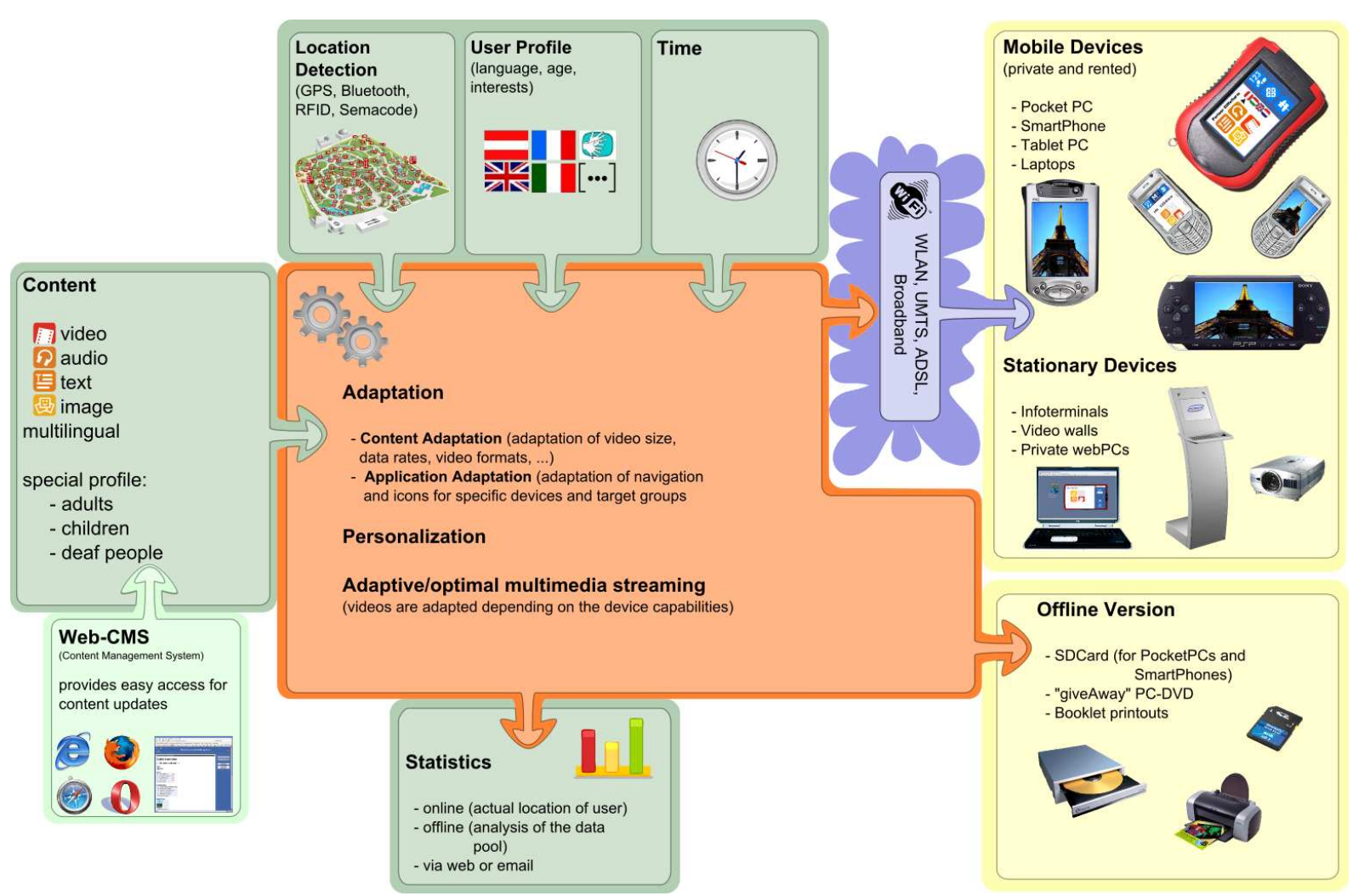

Figure 1: The big picture of the multimedia-based guidance system.

folder and booklet creation.

Finally, a statistics module logs complete user sessions and allows daily, weekly, or monthly statistics on, e.g., favorite/average content consumption, mainly used languages and profiles, average duration of sessions, and stopover times at certain objects of interest. In a networked scenario, the statistics are enhanced by online information about e.g. the number of active sessions, and the current locations of the users. This knowledge can easily be fed back into the adaptation system to offer improved content and presentation optimizations, and to give suggestions to the users.

\section{REFERENCE APPLICATION MULTIMUNDUS}

The M3-Guide is designed by having easy customization to different application areas like museums, fairs, theme parks, touristy regions, or even shopping centers in mind. As a first evaluation, our system was successfully introduced to Minimundus (Minimundus $\mathrm{GmbH}, 2006)$, an outdoor theme park in Klagenfurt, in June 2005.

Minimundus presents over 140 different 1:25scale replicas of world-famous buildings like the Eiffel Tower, or the Sydney Opera House. In allusion to "multimedia for Minimundus" we named the guidance application MultiMundus. In MultiMundus, all 140 exhibited objects are covered, and each object offers at least informative text stories and still images. 42 of them are enriched by audio and/or video stories, where a narrator gives in-detail information about these objects, fully enhanced by meaningful video scenes. All texts, audio, and video stories are available in four languages (German, English, Italian, and French). For deaf people, audio stories are also available as videos in Austrian sign language for these 42 objects.

The park is covered by a number of WLAN antennas, so rentable or private mobile devices can reach the main server. Ten rentable devices (see Figure 2) are available for a small fee. The devices are Linuxbased HP iPAQs 5550 with a ruggedized cover, headphones, a hand strap, and an input pen on a string.

The Minimundus team has access to the Webbased content management system and can easily manage the objects and their content. Live statistics 


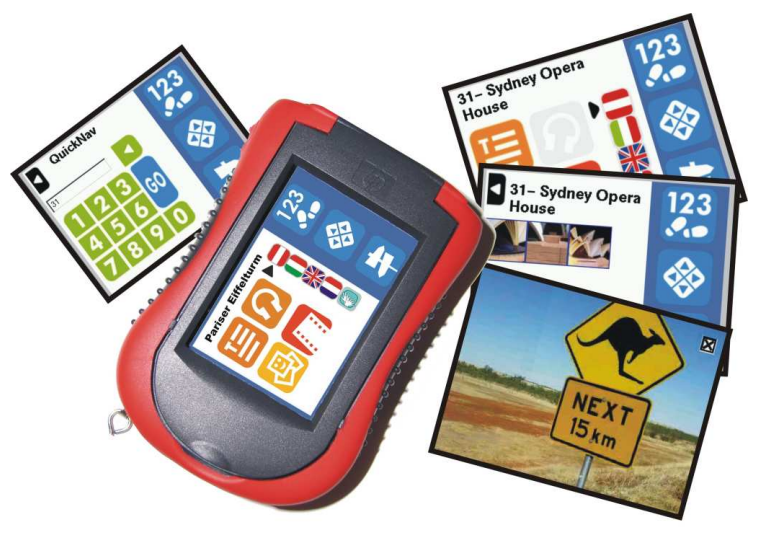

Figure 2: The rentable M3-Guide with screen shots.

about the actual usage of the guides are also available, monthly summarized statistics may be sent via email.

The Minimundus shop offers printed catalogues about the exhibited models in five different languages. Now, these catalogues are supplemented by PC DVDs, which provide the full MultiMundus presentation for later review on the home PC.

\section{SYSTEM MODULES AND TECHNICAL BACKGROUND}

\subsection{Content Management System}

The Web-based content management system (CMS) is designed for easy administration, and hence it is optimized for content administrators. First, it allows the insertion, editing, and deletion of profiles (like adults, children, and historical interest). With the chosen object oriented approach, it is possible to use inheritance and late binding between profiles. For example, there may be an adult profile, which inherits all objects and content to an inheriting profile, such as historical. It is possible to override content from inherited objects. In that case, a video for the adult profile would be replaced by a historically enhanced version. Of course, it is also possible to retain the video of the adult profile and to add an extra history video into the historical profile.

Second, the CMS enables for the insertion, editing, and deletion of object content with the following subtasks: editing the neighborhood, uploading videos, uploading audios, uploading images for the photo gallery module, and a built-in WYSIWYG editor for marked-up text content.

And third, built-in scripts automatically generate special info texts based on metadata. Consequently, each object has a standard text content showing the year of construction and the country of origin with a graphical flag.

For consistency reasons, the CMS works on a local copy of the repository when updating content. Therefore, it is possible to propagate changes when they are submitted, or to propagate them at a specific time when load on the live system is reduced (e.g., at night). For extendability reasons, the CMS is fully implemented by object oriented PHP scripts.

\subsection{Content Repository with XML Metadata}

The M3-Guide's repository is file-based (see Figure 3 ), hence it is easy to synchronize, to partially backup, and to update via shell scripts. It follows a simple structure, whereas each profile is represented by a folder, with all objects being folders again. In those object folders, an info.xml stores the object's title and other metadata about the object in all supported languages. In the MASTER folder, the different content types (audio, images, movies and stories) are managed. These subfolders hold either direct language versions for a single content, or - like in the stories folder - each story has its language version folders with an index.html there.

Besides the MASTER folder, each known device profile creates a subfolder with a cache of its transcoded and adapted content on demand. As a result, there are different video versions in different resolutions and codecs for different platforms, such as Flash video for the Windows-based Web PC and MPEG-4 video for Symbian-based smartphones.

\subsection{Transcoding Multimedia Cache}

Since the M3-Guide offers video and audio playback on many different consumer devices like Windows Mobile-based Pocket PCs, Linux-based PDAs, smartphones, and standard Web PCs, the content has to be transcoded and adapted to the consumer devices' capabilities. Since the content is mainly static, the transcoded content is also subject to caching. Moreover, many hand-held consumer devices are connected via expensive networks (as e.g., UMTS) on the one hand, and may be equipped with cheap memory cards on the other hand. Thus, a live-streaming of adapted content over expensive networks is not economic. Therefore, adapted content should be precached on local memory cards.

As a consequence, the M3-Guide uses a multilevel transcoding and caching architecture, where 


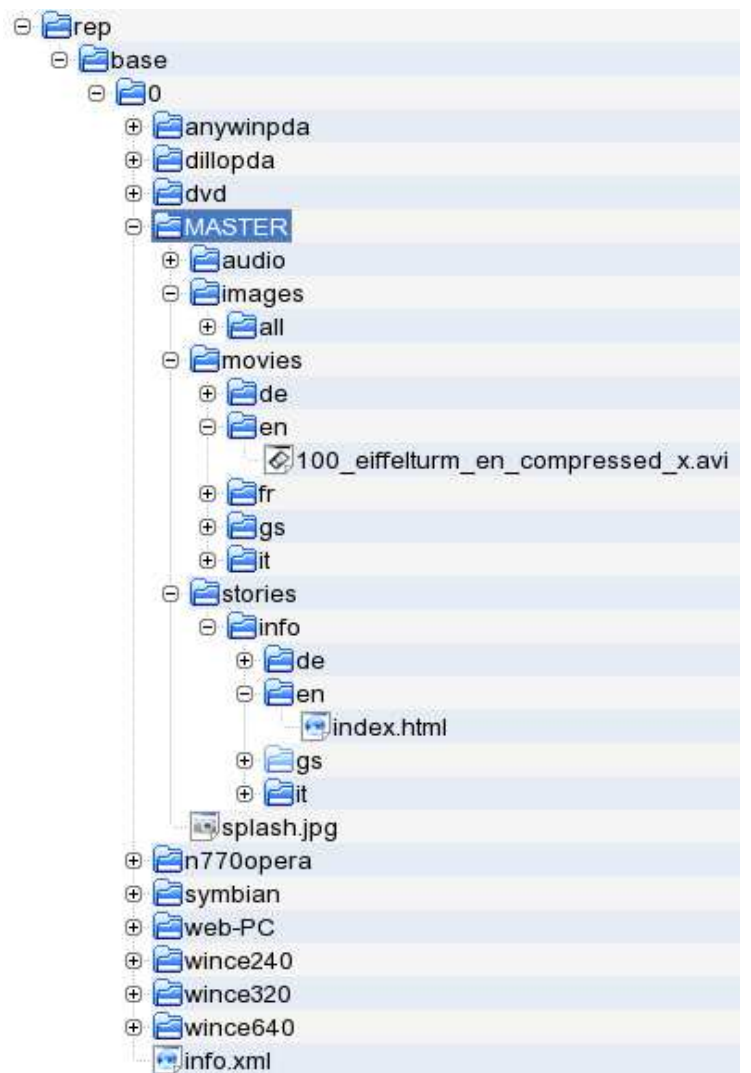

Figure 3: The content repository structure.

one or more master Transcoding Multimedia Caches (TMCs) run on the network and have direct access to the content repository via HTTP. On the consumer devices, a client media cache is implemented, which uses the local memory card as cache storage.

One of the master TMCs performs the transcoding step and, at the same time, stores the results in its server-side cache. Furthermore, it streams the transcoded data to the requesting consumer device, which in turn caches the adapted content locally. Consequently, later requests can be fulfilled from the local cache, and the master system and network is not used unnecessarily.

The TMC is written in platform-independent Perl and runs on Windows, Linux, and Windows Mobilebased platforms. Each TMC uses plugins for the known transcoding formats and caching behavior, depending on the underlying platform. Currently, combining all platforms, the following containers and codecs are supported: avi, wmv9, 3gp, $f v, s w f, m p g$, mp4, theora, mp3, ogg.

In principle, the requests to the TMC are HTTP URLs, and the device-specific parameters are coded into these URLs. If, for example, the real media

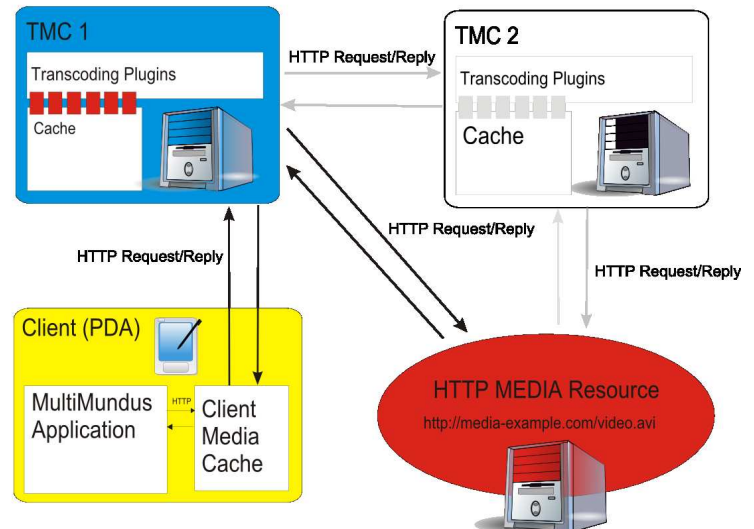

Figure 4: Schematic view of multiple transcoding multimedia caches.

was accessible from a Web server via http://mediaexample.com/video.avi (see Figure 4), a M3-Guide PDA would request this as a Windows Media video in $320 \times 240$ pixels via

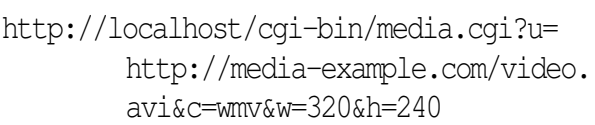

If the local TMC has no cached version stored, it will forward the request to the master TMC1 with the following URL:

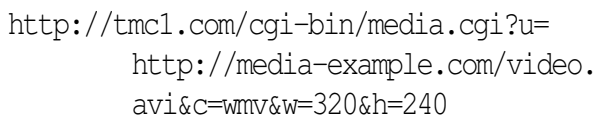

The master TMC1 may fulfill the request from its local cache, it may need to transcode the source of the video on its own, or it may ask another TMC2 for transcoding, if no suitable plugin is available.

\subsection{Statistics Module}

The statistics module manages the collected usage data and offers different views on them. When the mobile consumer devices have a permanent network connection, online statistics like the actual usage and the currently viewed object of a certain device are available.

Furthermore, offline interval statistics are calculated on a daily, weekly, or monthly basis and cover two groups of interests:

- data concerning the business: the number of visitors who used the system; cache capacity bottlenecks of rented devices; average device rental time; which objects/contents are frequently, which ones are rarely consumed; user demographics (language, age) 
- technical data, in order to improve the system itself: device management like maximum battery live time, battery recharge time, and irregular wear; click analysis of menu structures and navigation

The system performs a separation of information extraction queries, which are executed on the collected data, and of reports which prepare a business ready view for the management. These reports use textual and graphical output and are delivered as comma separated ASCII values (.csv), HTML, Latex, and PDF output. The reports are stored in an archive and sent to the responsible persons via email.

It is easy to dynamically integrate new queries and reports without the necessity of difficult system changes, and all queries and reports are dynamically parameterizable (e.g., it is easy to adjust the time intervals of reports).

Technically, the statistics module is implemented in PHP, initiated with cron jobs, and uses phplot for graphics creation.

\subsection{Server Details}

The M3-Guide server system uses Apache, PHP5.0, and MySQL to handle client connections, to process the repository, and to adapt the content to the current usage context.

The software is modularly written, so each content type like audio, video, image and story is handled by a special module, which itself is triggered by the request URL from the file-based repository.

When a content module claims responsibility for a certain content, it prepares a temporary XML representation of the application screen (e.g. the thumbnail view for images, or the text story), where also the content's metadata (like the title in the current language) is extracted from the corresponding info.xml. This XML output is then processed by an XSLT, which transforms the XML into the device specific HTML code for optimized presentation. This HTML code is cached and transmitted to the connected consumer device.

The MySQL database keeps track of currently connected sessions and their usage contexts. Thus, it also knows about the specifics of certain hardware device profile settings such as screen resolution or decodable video formats.

Finally, the M3-Guide offers a special exporter, which crawls through the repository and prepares a device-specific HTML representation of all available profiles and objects including their contents. These local dumps are enhanced by device-specific applications like an auto starter or a self-extracting Web browser. These dumps can be distributed on SDCards and DVDs, which may be used on private Pocket and Home PCs, respectively.

\subsection{Supported Mobile Devices}

\subsubsection{Pocket PCs with Windows Mobile}

For Windows Mobile-based Pocket PCs we have developed a $\mathrm{C \# /.net} \mathrm{application,} \mathrm{which} \mathrm{offers} \mathrm{a} \mathrm{full-}$ screen object presentation by the use of a Pocket Internet Explorer widget and custom video player. All hardware buttons are reprogrammed and we offer a library to access the built-in WLAN, GPS, and Bluetooth. The HTML content is delivered either locally from an SD-Card or via WLAN. Additionally, a local media cache (see Section 5.3) delivers all videos from the local SD-Card or via WLAN. The navigation either works manually via the Quick Navigation (by entering object numbers), or automatically via GPS and Bluetooth, if applicable (Santner et al., 2006).

The Windows Mobile-based guidance application can be stored onto an SD-Card, as it is the case for videos and all other content. After plugging in the SD-Card into a tested Pocket PC, the M3-Guide application is started by the operating system automatically.

\subsubsection{Pocket PCs with Linux}

For the reference application MultiMundus, we used Linux-based HP iPAQs 5550 with Familiar Linux, a well-patched Dillo Web browser, and our own video player based on our self-developed multimedia library ViTooKi (Kropfberger and Schojer, 2003). The boot process was modified in a way that the system always boots into full-screen mode, without a window manager, and without any functionality of hardware buttons. Due to performance reasons, the rentable devices use a memory card for the local caching of videos. Thus, the WLAN is not bothered too much with parallel video streaming. All presentation data and not yet cached videos are retrieved via WLAN from the server.

\subsubsection{Smartphones with Symbian OS}

We have ported the M3-Guide to Symbian 8.0s Series 60 2nd Edition, shipped on an RS-MMC dual voltage memory card. Since the system is completely HTTPbased, it is easy to connect via UMTS and hereby spare the local MMC card. This may offer more upto-date information, but will lead to high telephone costs. 
Our software launches an Opera Web browser and then behaves like a Web server, which offers the local MultiMundus content. When a video is requested, our own full-screen video player is started. Videos are stored as 3gpp containers including MPEG4 video and AAC audio streams. The video is resized to $176 \times 208$ pixels and transcoded to the correct codecs by our automatic server-side adaptation process. See the two screenshots of a Nokia 6630 smartphone in Figure 1 . The navigation is realized by manually entering object numbers and is handled with a HTTPCGI application.

Another useful feature is the server-side interpretation of specially formed HTML links which describe telephone numbers. After clicking such a link, the Symbian smartphone starts to dial the number. This enables for a personalized booking of certain services.

\subsubsection{Further Devices}

The M3-Guide was successfully tested with various WLAN-enabled devices such as the Nokia N770 Internet Tablet or the Sony Playstation Portable. After connecting to the Multimundus WLAN ESSID, it was possible to request the HTML start page via the built in Web browsers. Video and audio were played back via built-in players. Of course, the presentation did not perfectly fit the screen resolutions and other device capabilities like optimized video codecs, but at least it was usable out of the box.

\subsubsection{Supported Usage Scenarios}

\begin{tabular}{|l||l|l|l|}
\hline Device profile & $\begin{array}{l}\text { network } \\
/ \text { un- } \\
\text { touched }\end{array}$ & $\begin{array}{l}\text { network } \\
/ \text { pre- } \\
\text { pared }\end{array}$ & $\begin{array}{l}\text { local } \\
\text { pare- }\end{array}$ \\
\hline \hline $\begin{array}{l}\text { iPAQ h5550 with } \\
\text { Embedded Linux }\end{array}$ & & $\checkmark$ & $\checkmark$ \\
\hline $\begin{array}{l}\text { Pocket PC with } \\
\text { Windows Mobile }\end{array}$ & $\checkmark$ & $\checkmark$ & $\checkmark$ \\
\hline $\begin{array}{l}\text { Smartphone with } \\
\text { Symbian OS }\end{array}$ & & $\checkmark$ & $\checkmark$ \\
\hline $\begin{array}{l}\text { Nokia 770, } \\
\text { Playstation } \\
\text { Portable }\end{array}$ & $\checkmark$ & & \\
\hline $\begin{array}{l}\text { WebPC / Info ter- } \\
\text { minal / DVD }\end{array}$ & $\checkmark$ & $\checkmark$ & $\checkmark$ \\
\hline
\end{tabular}

Table 1: Supported platforms and devices

Table 1 gives an overview of all supported platforms and devices, and classifies the support based on three possible usage scenarios.
The first column depicts the ideal scenario, where a private (untouched) device may access our M3Guide system via a configured network without any software installation process. We support this for Windows Mobile-based devices, private Web PCs, and to some extent (see previous subsection) for the Nokia 770 and the Sony Playstation Portable.

The two other columns discuss well-prepared devices, where optimized video players are installed and a custom application is presented in a fullscreen kiosk mode. The content is either retrieved via a network, or from a local storage subsystem. In order to reduce future network load, some devices use a hybrid approach where content is cached on a local storage subsystem.

\section{USAGE ACCEPTANCE AND FACED PROBLEMS}

The M3-Guide's reference application MultiMundus (see section 4) was introduced to Minumundus Klagenfurt in June 2005, and was available there to visitors during the whole remaining operating year 2005 (about four months until the end of October). This time period was used to evaluate the usage acceptance and performance behaviour of the guidance system, and to consequently improve the system based on the obtained evaluation results.

Summarizing the usage evaluation results, such a location-aware and multimedia-enhanced guidance system is rather a high-end solution for some interested visitors, who want to consume more information than provided by commonly available catalogues and brochures. For example, in the most frequent month August, 123 out of about 40.000 visitors rented the MultiMundus guide, resulting in a rental percentage of only $0,31 \%$. Since there were ten mobile guides available for rent, and each device was rentable about three times each day (due to usage and battery recharging times), the actual rentals also reached only $13,23 \%$ of 930 possible rentals. Thus, two rentable mobile devices would have been enough on average.

The reasons for these low rental rates are manifold. First, Minimundus did not offer the MultiMundus guides directly at the cash. Instead, the place of disbursement was separated to the main entry in the park. Second, Minimundus also offered "old" audio guides in parallel to our MultiMundus guide. Some people just wanted to passively listen to spoken text, rather than to actively read or watch videos. Third, the MultiMundus guide was more difficult to explain than it was the case with the audio guides, although the visitors found that the usability of the MultiMundus 
guide was good. As a consequence, the staff responsible for handing out these devices got more and more lazy with explaining the MultiMundus guide's usage, and preferred to hand out the old audio guides. And finally, the mobile guides were encapsulated in rugged cases to prevent them from damages on occasional drops and rains. The rugged cases made the guide considerably larger and more unhandy to wear.

Beside the low rental rate problem, there were two more crucial problems related to the used mobile devices. First, the battery life cycles where partially really short. Many of the used batteries were dead at the end of the season. And second, the staff who handed out the devices has occasionally broken some of the power connectors, when plugging them into the slideon receptacles. Sometimes, even the hardware reset buttons were pushed through and hence those devices had to be repaired.

\section{CONCLUSION}

A Web-based and multimedia-enhanced guidance system has been introduced that customizes both content and its presentation to the current usage context. Content may include different coding and presentation formats like video, audio, image, and marked-up text, and can be managed by the means of a Webbased content management system. Presentations are based on standard HTML documents for images and texts, and on custom and commonly pre-installed video players for video and audio streams. The usage context plays a central role in the whole adaptation process. It spans a number of different dimensions, including the user profile, the consumer device capabilities, the network capabilities, the location, and the time of content consumption.

The presented guidance system was introduced and evaluated in a local outdoor theme park in 2005 , and was available to visitors of this park over the whole operating year. Statistical evaluations have shown that such a multimedia-enhanced guide is rather a high-end solution for some visitors, who want to consume more content than provided by commonly available catalogues.

\section{REFERENCES}

Abowd, G. D., Atkeson, C. G., Hong, J., Long, S., Kooper, R., and Pinkerton, M. (1997). Cyberguide: A Mobile Context-aware Tour Guide. Wireless Networks, 3(5):421-433.
Bormans, J. and Hill, K. (2002). MPEG-21 Overview v.5. ISO/IEC JTC1/SC29/WG11/N5231.

Bra, P. D. and Calvi, L. (1998). AHA! An Open Adaptive Hypermedia Architecture. The New Review of Hypermedia and Multimedia, 4:115-140.

Brusilovsky, P. (1996). Methods and Techniques of Adaptive Hypermedia. User Modeling and User-Adapted Interaction, 6(2-3):87-129.

Bulterman, D., Grassel, G., Jansen, J., Koivisto, A., Layaïda, N., Michel, T., Mullender, S., and Zucker, D. (2005). Synchronized Multimedia Integration Language (SMIL 2.1). W3C Recommendation.

Devillers, S., Timmerer, C., Heuer, J., and Hellwagner, H. (2005). Bitstream Syntax Description-Based Adaptation in Streaming and Constrained Environments. IEEE Transactions on Multimedia, Special Issue on MPEG-21, 7(3):463-470.

Klyne, G., Reynolds, F., Woodrow, C., Ohto, H., Hjelm, J., Butler, M. H., and Tran, L. (2004). Composite Capability/Preferences Profile (CC/PP): Structure and Vocabularies 1.0. W3C Recommendation.

Korva, J., Plomp, J., Määttä, P., and Metso, M. (2001). Online Service Adaptation for Mobile and Fixed Terminal Devices. In IEEE 2nd International Conference on Mobile Data Management (MDM) 2001, pages 252259, Hong Kong, China.

Kropfberger, M. and Hellwagner, H. (2005). Combining Stream Switching with Fine-grained Intra-stream Adaptation for Adaptive Video Streaming. In IEEE 7th International Workshop on Multimedia Signal Processing (MMSP) 2005, pages 373-376, Shanghai, China.

Kropfberger, M. and Schojer, P. (2003). ViTooKi - The Video ToolKit. Open source project at SourceForge.net. http://ViTooKi.sourceforge.net

Krösche, J., Baldzer, J., and Boll, S. (2004). MobiDENK Mobile Multimedia in Monument Conservation. IEEE MultiMedia, 11(2):72-77.

Lemlouma, T. and Layada, N. (2003). SMIL Content Adaptation for Embedded Devices. In SMIL Europe 2003 , Paris, France.

Lemlouma, T. and Layada, N. (2004). Context-Aware Adaptation for Mobile Devices. In IEEE 5th International Conference on Mobile Data Management (MDM) 2004, pages 106-111, Berkeley, USA.

Minimundus GmbH (2006). Die kleine Welt am Wörthersee. http://www.minimundus.at

Open Mobile Alliance (2006). User Agent Profile Approved Version 2.0. OMA-TS-UAProf-V2_020060206-A.

Santner, M., Tusch, R., Kropfberger, M., Böszörmenyi, L., and Hellwagner, H. (2006). Ein Ortserkennungssystem für mobile Touristenführer. In DACH Mobility 2006, pages 84-98, Ottobrunn, Germany.

Scherp, A. and Boll, S. (2005). Managing Multimedia Semantics, chapter 11: MM4U: A Framework for Creating Personalized Multimedia Content, pages 246-287. Idea Group Inc. 
Schojer, P., Böszörmenyi, L., Hellwagner, H., Penz, B., and Podlipnig, S. (2003). Architecture of a Qualitybased Intelligent Proxy (QBIX) for MPEG-4 Videos. In ACM 12th International World Wide Web Conference, pages 394-402, Budapest, Hungary.

Timmerer, C. and Hellwagner, H. (2005). Interoperable Adaptive Multimedia Communication. IEEE Multimedia Magazine, 12(1):74-79.

Vetro, A. and Timmerer, C. (2005). Digital Item Adaptation: Overview of Standardization and Research Activities. IEEE Transactions on Multimedia, 7(3):418426.

Vetro, A., Timmerer, C., and Devillers, S. (2006). The MPEG-21 Book, chapter 7: Digital Item Adapation - Tools for Universal Multimedia Access, pages 243280. John Wiley and Sons Ltd. 